\title{
Le logmètre : un nouvel outil de saisie des descriptions de carottes
}

\author{
Bruno SAVOYE, Gilbert FLOCH, René KERBRAT, Ronan APPRIOUAL
}

Ifremer, BP 70, 29280 Plouzané, France

(Reçu le 14 janvier 1997, révisé le 29 août 1997, accepté le 16 octobre 1997)

\begin{abstract}
The logmeter: a new core measuring board based on bar codes. Core description is used extensively by sedimentologists to yield critical information from sediments. It consists of a naturalistic description of the visual and textural characteristics of the sediment. It is generally a manual procedure. However, digital core descriptions are now required to manage data processing. Hand-written description can bc kcycd into a computer, but it is tedious and timc consuming. Moreover, recording and typing errors can occur. A system is presented for digital description of sediment cores using a bar code principle. It can be used in the laboratory as well as on board vessels. It comprises a box inside which the half-core can be placed. A ruler and several sets of bar codes are printed on the inside box top. Bar codes are read by a waterproof sensor pencil that is interfaced with a PSION $3 \mathrm{~A}$ pocket computer. The software mimicks the traditional method of core description, the geologist answering questions using the optical pencil. It offers versatility and is designed with the geoscientist's need for flexibility and ease of use in mind. It allows computerised core data management and is highly upgradable. It can help to harmonise core description and to compare it with core data provided by the new multiparameter measurement systems. Detailed log plots no longer require tedious coding. Logmeter data can be used to automatically create customized, full-featured log graphs displaying lithology, descriptions, curves, histograms, graphic symbols and attractive legends, using commercial or dedicated softwares. () Elsevier, Paris / Ifremer / Cnrs / Ird
\end{abstract}

\section{core / bar codes / sediment / measuring board / software processing}

Résumé - Le logmètre est un outil de saisie de données basé sur l'utilisation de codes-barres. Il peut être utilisé indifféremment à bord des navires ou en laboratoire pour la description des carottes de sédiments. Le système se présente comme un boîtier à l'intérieur duquel on place la demi-carotte à décrire. Le couvercle intérieur du boîtier comporte une règle graduée et différents ensembles de codes-barres qui sont lus au moyen d'un stylo laser interfacé avec un ordinateur de poche de type Psion 3A. L'ordinateur est programmé pour reproduire une séquence de description type. L'opérateur répond aux questions en utilisant soit le crayon optique, soit le clavier. Ce système simple et évolutif permet d'harmoniser les descriptions de carottes en vue de leur traitement informatique et apporte un gain de temps et de fiabilité par rapport à la procédure manuelle classique. (C) Elsevier, Paris / Ifremer / Cnrs / Ird

\section{carotte / codes-barres / sédiment / outil / traitement informatique}

\section{INTRODUCTION}

Le géologue qui décrit des carottes effectue un travail essentiel d'acquisition de données de base. Ce travail pourrait surprendre un physicien habitué à la rigueur des équations et au raisonnement logique, dans la mesure où il est extrêmement naturaliste. Le sédimentologue doit en effet décrire la carotte, en faire l'inventaire visuel méthodique, rigoureux et complet sans se perdre dans les détails non significatifs et en chassant au mieux imagination, subjectivité et interprétation.

Cette description, qui a pour objectif la mise en évidence des caractéristiques visibles des sédiments (nature, texture, structures, géométrie, couleur, limites...), doit permettre l'interprétation des sédiments en termes de processus ou de stratigraphie, après confrontation avec des données complémentaires comme les mesures physi- 
ques ou les images géophysiques (sonar, sismique). Comme toute acquisition de données, la description doit être aussi objective que possible afin d'être réutilisable par des tierces personnes ou après plusieurs années, même si les concepts scientifiques ont changć. C'est un travail systématique, généralement manuel, souvent long et fastidieux. Le résultat final dépend beaucoup de la compétence et de l'expérience du descripteur.

Depuis quelques années, le développement de plusieurs techniques d'investigation sur carottes a considérablement modifié le travail du sédimentologue, ouvrant le champ au développement de véritables techniques de diagraphies sur carottes : bancs multiparamètres (gammadensité, susceptibilité magnétique, gamma-ray, vitesses des ondes $\mathrm{p}$, etc.) et plus récemment, bancs vidéo ou bancs de radio $\mathrm{X}$ numériques.

Malgré ces développements, la description visuelle des carottes par un géologue demeure un passage obligé. Dans les laboratoires de recherche qui ne sont pas équipés des outils de nouvelle génération, ou dans les sociétés de service qui ont souvent à décrire de très nombreuses carottes sur chantier et dans un temps limité, la description visuelle est encore souvent la seule véritable information de base sur les sédiments. Pour les groupes mieux équipés, la description visuelle reste un élément indispensable pour l'intégration des données issues des différents bancs de mesure. C'est pourquoi la description des carottes représente, et représentera encore à moyen terme, une part importante du travail des sédimentologues en général, et des sédimentologues marins et lacustres en particulier. Le développement des longs carottiers au cours des dernières années, ainsi que la mise en place de nouveaux programmes de recherche basés sur l'échantillonnage quasi continu de la colonne sédimentaire (programme européen Corsaires, Geofrance 3D, programme Images) engendre d'ores et déjà l'analyse d'un nombre croissant de carottes.

Devant l'accroissement considérable du volume des informations à traiter et afin de mieux comparer les descriptions de carottes et les données issues des bancs de mesures, la description sur papier est souvent saisie sur ordinateur. Elle peut ainsi être stockée, traitée et alimenter des bases de données ou d'informations géographiques, mais la saisie au clavier est fastidieuse et source d'erreurs de frappe ou de relecture.

En 1991, le service des pêches d'Ifremer-Brest a développé et breveté un système de prise de mensurations de poisson, baptisé ichtyomètre [2]. Basé sur l'utilisation de codes-barres, il permet une saisie rapide et performante des caractéristiques des poissons, à bord des bateaux de pêche ou dans les criées. Plusieurs exemplaires de ce système sont actuellement en service dans différents pays curopéens.

Partant de ce principe et par extension du concept, nous avons entrepris de développer un outil de saisie des descriptions de carottes baptisé logmètre. Le prototype a été testé en septembre 1995 [1] et a bénéficié de plusieurs améliorations.

\section{DESCRIPTION DU LOGMÈTRE}

Le logmètre comprend deux parties distinctes : un boîtier, un ordinateur de poche (Psion 3A) interfacé avec un crayon optique.

Le logmètre est basé sur le principe de lecture et d'enregistrement de codes-barres. La partie électronique est complètement indépendante du bôttier et ne comprend que des éléments standards et commercialisés. Elle pourrait donc être mise à niveau au fur et à mesure des évolutions techniques.

\subsection{Boîtier}

Le boîtier du logmètre est en duralinox et mesure $21 \mathrm{~cm}$ de large pour $100 \mathrm{~cm}$ de long. Fermé, ce boîtier permet le transport de l'ordinateur, du crayon optique et du petit matériel de laboratoire. Lors des descriptions, l'utilisateur dispose la carotte ouverte à l'intérieur du boîtier, couvercle ouvert et rabattu sur le côté. Sur la face intérieure du couvercle sont imprimés plusieurs panneaux de codes-barres (figure 1). Un jeu de cales permet de travailler sur des carottes de diamètre variant de 6 à $15 \mathrm{~cm}$. Pour les carottes d'une longueur supérieure à $1 \mathrm{~m}$, il est possible d'utiliser une rallonge de $50 \mathrm{~cm}$.

Le couvercle comporte cinq panneaux de codes-barres : la règle de mesure, un panneau alphanumérique, un panneau de caractérisation texturale, un panneau de description des structures et enfin un panneau de description des figures sédimentaires (figure 2). Chaque code-barres est flanqué d'un code machine de deux ou trois lettres et d'une légende explicite (voir liste tableau I). La feuille imprimée qui contient les codes-barres est collée et protégée par une feuille en polycarbonate. En cas de détérioration, la feuille des codes-barres, voire uniquement sa couverture transparente en plastique, peuvent être chan- 


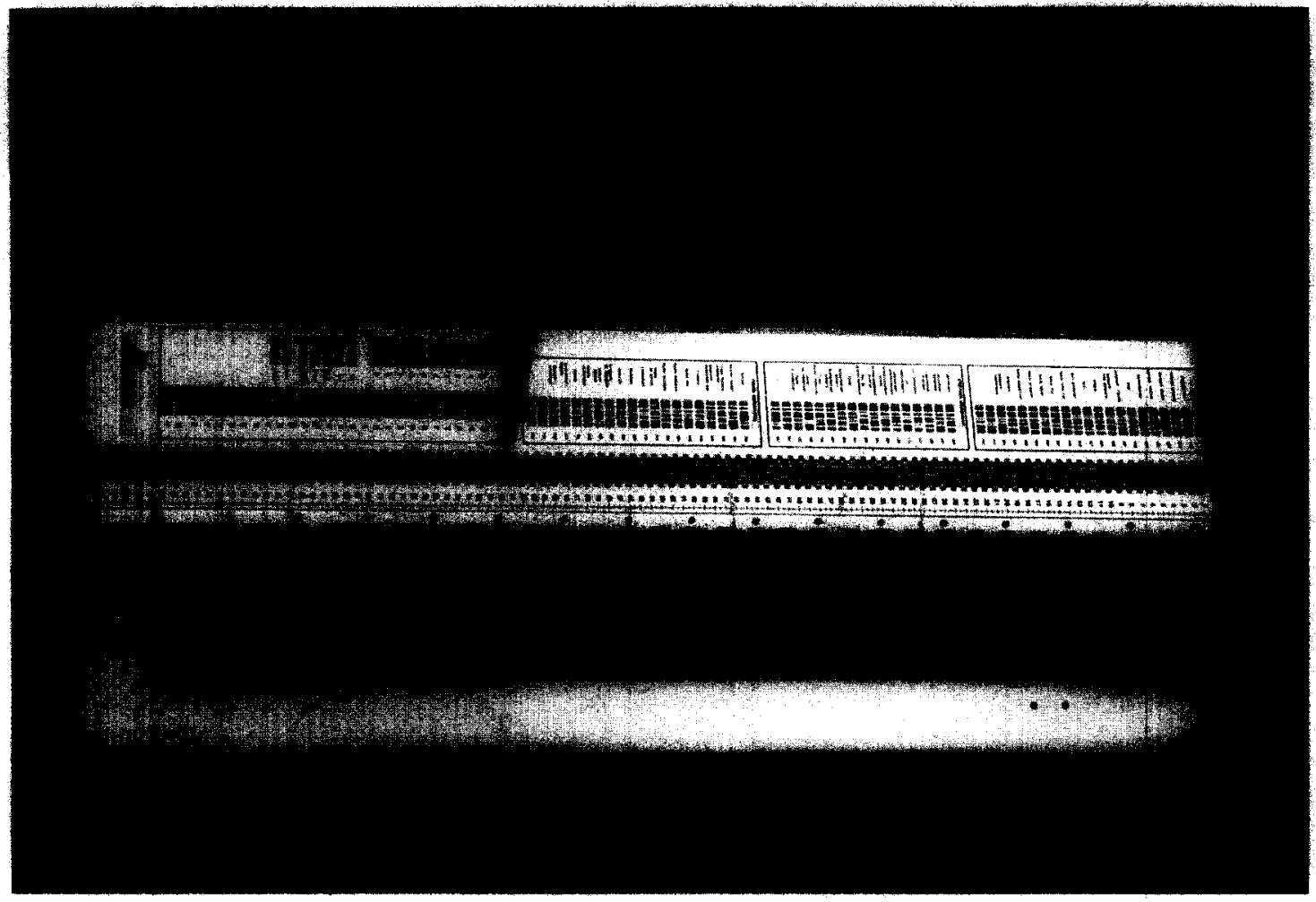

Figure 1. Photo du logmètre dans son ensemble avec le boîtier, le crayon optique, et l'ordinateur de poche. Le boîtier reçoit la demi-section de carotte à décrire. Une règle graduée et cinq panneaux de codes sont sérigraphiés sur la face intérieure du couvercle. Noter que chaque codebarres est accompagné de sa légende en clair.

Figure 1. Photograph of the complete logmeter system including the board, the waterproof sensor pencil and the pocket computer. The logmetre itself combines a box for core section and a ruler and five bar code pads arranged on the inside cover. Note that the explanations of the bar codes are available.

gées sans qu'il soit nécessaire de remplacer tout le système.

La règle de mesure comporte une règle, graduée tous les demi-centimètres, équipée de codes-barres pour la prise de cote. Pour une précision inférieure au demi-centimètre, la valeur exacte d'une cote est lue sur la règle graduée et est entrée dans l'ordinateur en utilisant, soit le clavier, soit les codes-barres du panneau alphanumérique.

Le panneau alphanumérique comporte les lettres de l'alphabet et les chiffres, ainsi que quelques fonctions telles que « effacer », « retour », « oui », « non ». Il est donc possible d'entrer des commentaires dans l'ordinateur sans utiliser le clavier.

Le panneau de caractérisation texturale comprend 18 codes qui permettent de décrire la granulométrie du sédiment tels que « silt grossier » ou « sable moyen » et les limites, ainsi que la nature des limites tel que « contact érosif».
Le panneau de description des structures sédimentaires comprend 15 codes, tels que « lamination », « granoclassement normal » ou « très bioturbé ».

Le panneau de description des figures et composants sédimentaires comprend 18 codes, tels que « bioclast», « cendre volcanique » ou « débris coquilliers ».

Chacun de ces trois derniers panneaux comporte deux codes libres supplémentaires. Ils peuvent être utilisés pour prendre en compte des caractéristiques peu courantes observées dans une carotte ou dans un ensemble de carottes. C'est la garantie de l'adaptabilité de l'outil à l'utilisateur ou à la carotte à décrire.

\subsection{Crayon optique}

Le crayon optique scrt à lire les codes-barres et assure l'interface entre le boîtier et l'ordinateur. Il est étanche et peut être acheté dans n'importe quel magasin spécialisé. 


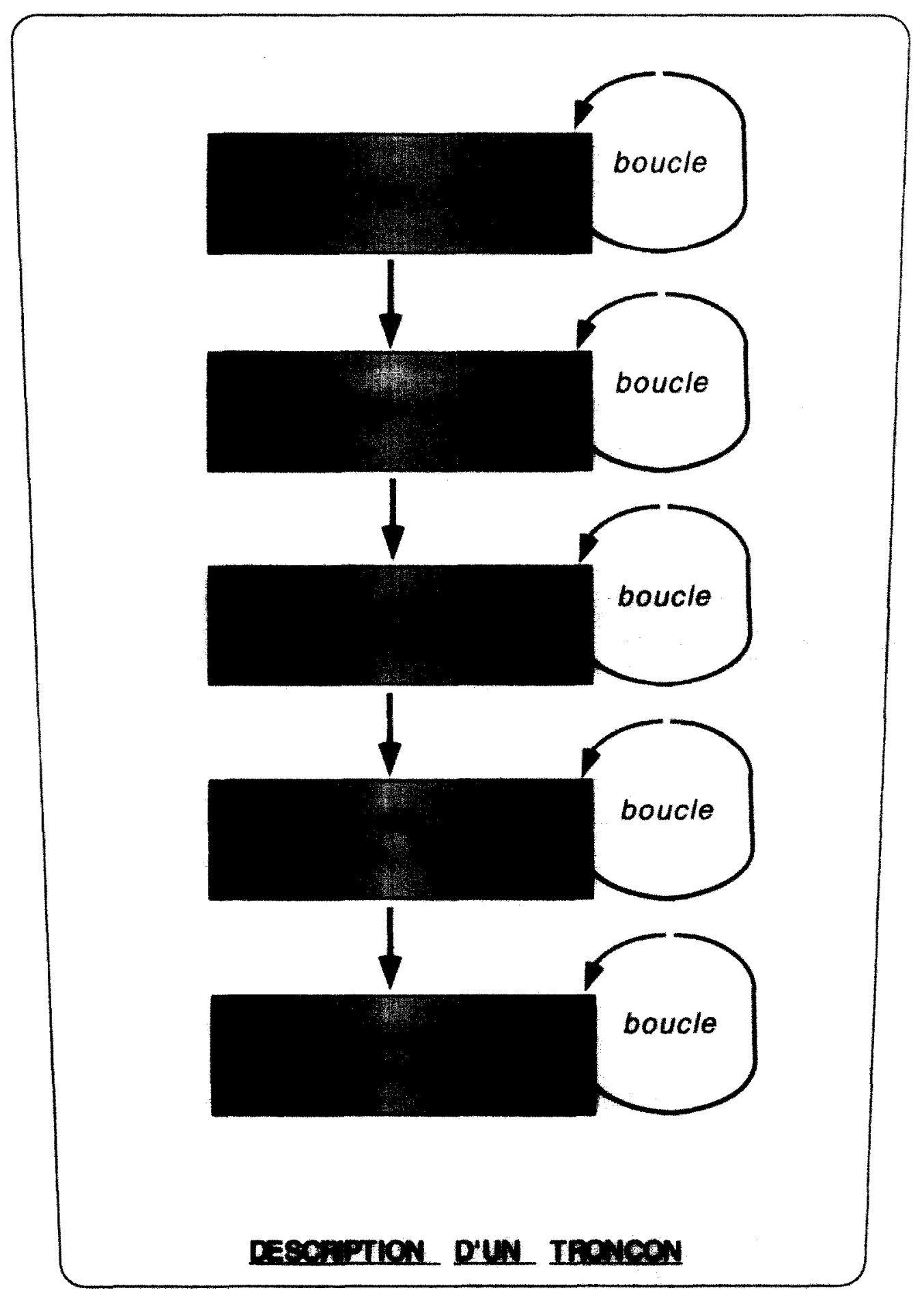

Figure 2. Synoptique du logiciel.

Figure 2. Logmeter software synoptic. 
Tableau I. Liste des codes-barres disponibles par panneau.

Table I. $I$ ist of available har codes.

\begin{tabular}{llllll}
\hline Contact net & CTN & Lamination & LMA & Terrier & TER \\
Contact graduel & CTG & Lamination texturale & LMT & Terrier ouvert & TRO \\
Contact érosif & CTE & Lamination chimique & LMC & Trace fossile zoophycos & ZOP \\
Contact incliné & CTO & Lit convolute & LCV & Cheminée d'expulsion d'eau \\
Perturbation & PRT & Lit lenticulaire & LTL & Fissure & FIS \\
Vide & VID & Laminations entrecroisées & LME & Galet mou & GLM \\
Sédiment fluide & SDF & Granoclassement normal & GRN & Poche sableuse & POS \\
Sédiment très compacté & SDC & Granoclassement inverse & GRI & Poche silteuse & PSI \\
Argile & ARG & Pas de granoclassement & GRP & Poche de pyrite & PYT \\
Silt & SLT & Slump & SLP & Lithoclast & LTC \\
Silt grossier & SLG & Bioturbation majeure & BRM & Bioclast & BCT \\
Sable très tin & STF & Bioturbation mineuse & BRT & Coquille & COQ \\
Sable fin & SBF & Front d'oxydo-réduction & FOX & Fragment de coquille & VOF \\
Sable moyen & SBM & Tache de réduction & FCR & Débris ligneux & DBL \\
Sable grossier & SBG & Tache d'oxydation & TCO & Cendre volcanique & CVQ \\
Sable coquillier & SBQ & Code libre 1 & SS1 & Corail & CRL \\
Graviers & GRV & Code libre 2 & SS2 & Concrétion/Nodule & CCT \\
Sédiment indéterminé & STI & & & Matière organique \\
code libre 1 & PT1 & & & Code libre 1 \\
code libre 2 & PT2 & & & Code libre 2 \\
\hline
\end{tabular}

\subsection{Ordinateur et logiciel}

L'ordinateur de type Psion 3A, auquel est connecté le crayon optique est peu encombrant $(16 \times 12 \times 2 \mathrm{~cm})$ et ultra-léger $(325 \mathrm{~g})$. Il a une autonomie de $80 \mathrm{~h}$ avec des batteries rechargeables, un écran de huit lignes pleines et un haut-parleur incorporé avec possibilité de sonnerie.

\subsubsection{Procédure de description}

L'ordinateur est programmé afin de reproduire aussi fidèlement que possible la procédure de description d'une carotte standard. Il sert d'aide-mémoire en posant une série de questions conventionnelles («nature du sédiment ? » par exemple) auxquelles l'opérateur répond en lisant le code-barres adéquat avec le crayon optique. Un bip sonore ponctue l'acquisition de chacune des réponses. Les questions posées par l'ordinateur reconstruisent pas à pas la séquence de description traditionnelle suivie par le géologue et les codes-barres disposés sur le logmètre permettent de répondre à tous les types de questions : cote de la limite, nature de la limite, couleur du sédiment, granulométrie moyenne observée, type de sédiment, cote de la prise d'échantillon, etc. Les données saisies peuvent être corrigées à n'importe quelle étape de la séquence.

La procédure proposée pour décrire les carottes est celle en usage à Ifremer, très proche de celle pratiquée par d'autres laboratoires de géologie marine en France ou à l'étranger. La description est réalisée tronçon par tronçon. Elle comporte quatre parties distinctes qui tournent en boucle tant que l'opérateur le désire : saisie des codes et limites de couleur, saisie des caractéristiques texturales du sédiment, description des structures sédimentaires, description des composants sédimentaires ou de paramètres ponctuels, saisie des prises d'échantillons (figure 2).

\subsubsection{Transfert et utilisation des données}

À partir des ordinateurs Psion, les données peuvent être très facilement transférées sur PC ou Mac (figure 3) ct être exploitées en utilisant des logiciels standard de base de données (tableau II). Nous utilisons pour l'instant une application écrite sous Foxpro pour la visualisation des logs et l'interprétation comparée avec les données complémentaires telles que gammadensité, photo des carottes ouvertes, radio $X$, teneur en carbonates, etc. (figure 4). Il est possible de visualiser les données en utilisant des logiciels de logplotting disponibles dans le commerce.

\section{AVANTAGES ET LIMITES DU LOGMÈTRE}

Par rapport au mode de description traditionnel, l'utilisation du logmètre présente plusieurs avantages, dont un gain de temps non négligeable et une réduction des coûts. Les erreurs de frappe, de lecture d'un texte manuscrit, ou 


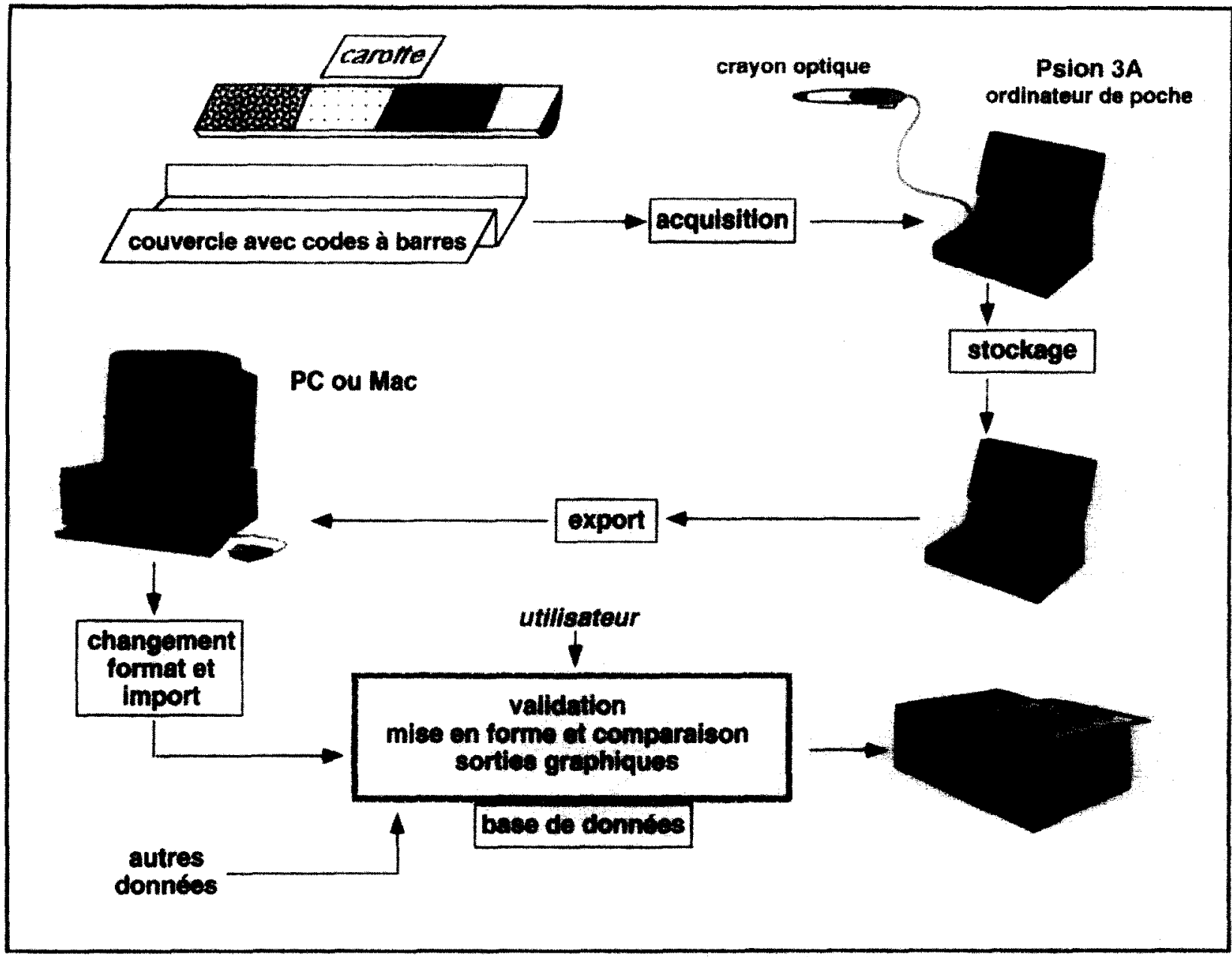

Figure 3. Schéma de la procédure de traitement des données.

Figure 3. Logmeter data processing scheme.

de compréhension sont évitées et les données sont saisies rapidement sous une forme directement utilisable par n'importe quel type d'ordinateur personnel $[3,4,5]$.

L'avantage du logmètre sur d'autres systèmes de saisie électronique tient à son faible coût de revient et surtout à sa capacité à travailler en environnement humide sans risque d'inondation ou de corrosion. Il évite toute opération d'écriture et de report manuel. Il est donc adapté aux conditions de travail en laboratoire à terre ou en mer.

Enfin il est, par construction, très ouvert et évolutif, dans la mesure où le boîtier et la partie électronique sont parfaitement indépendants.

Ce système a des limites. S'il a été conçu afin de permettre la description d'une très grande variété de carottes, le fait qu'il impose un mode de description des carottes, est limitant. Dans la mesure où deux codes-barres libres par panneaux sont disponibles, la limitation réside dans la programmation de la procédure. Rien n'interdit de reprogrammer l'ordinateur en fonction de besoins spécifiques à un groupe d'utilisateurs tout en conservant la partie mécanique du logmètre, voire de disposer sur la même base matérielle de différents modes de description. Il convient de préciser que, dans sa version actuelle, le logmètre ne peut pas rendre compte de surfaces inclinées ou très irrégulières (failles, bases de bancs, etc. figure 4).

Le choix préalable d'une procédure de description est nécessaire. Il présente l'avantage d'aider à la standardisation et donc à l'homogénéisation du mode de description 


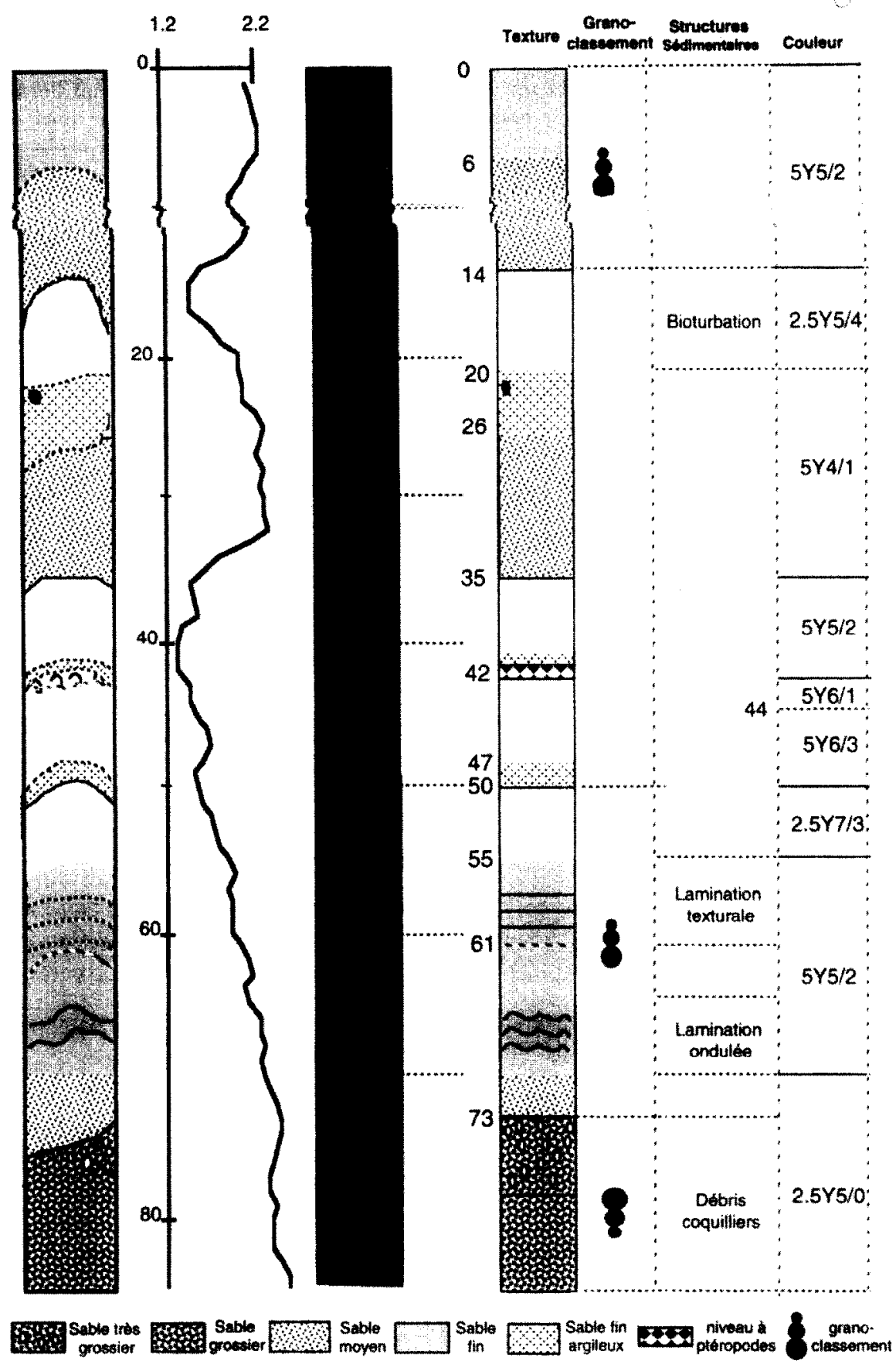

Figure 4. Exemple de sortie des données collectées sur une carotte avec le logmètre, comparées aux données acquises de manière plus classique. De gauche à droite : log synthétique dessiné à la main (procédure classique), courbe de Gamma-densité issue du passage au banc de mesure multiparamètres, photo de la carotte ouverte, $\log$ et description de la carotte réalisés de manière pseudo automatique à partir des données numériques issues du logmètre (voir tableau $I I$ ).

Figure 4. Example of a core log-plot using logmeter collected data, compared with more conventional data. From left to right: Hand-drawn $\log$ of the core, gamma-density curve from multiparameter measuring board, core photo, and core log and descriptive parameters automatically displayed using logmeter collected data. 
Tableau II. Exemple de tìchier collecté avec le logmètre sur une carotte prélevée par $2600 \mathrm{~m}$ de profondeur d'eau au large de la Corse. Le fichier est de taille réduite car la structure de la carotte est simple. Un log synthétique de la carotte est présenté en figure 4.

Table II. Example of data file collected using the logmeter and for a core collected at $2600 \mathrm{~m}$ water depth off Corsica (western Mediterranean). The file size is small due to the relatively simple core structure. A synthetic log of the core is shown in figure 4.

\begin{tabular}{|c|c|c|c|c|c|c|c|c|}
\hline descripteur & prélèvement & section & sommet & base & limite-séd & texture & zonaux & commentaire \\
\hline B. Savoye & KNI 06 & 1 & 0 & 6 & CTN & $\mathrm{SB} \Gamma$ & & \\
\hline B. Savoye & KNI 06 & 1 & 6 & 14 & $\mathrm{CTI}$ & SBM & GRN & \\
\hline B. Savoye & KNI 06 & 1 & 14 & 20 & CTN & ARG & & \\
\hline B. Savoye & KNI 06 & 1 & 20 & 26 & CTN & SBF-ARG & & \\
\hline B. Savoye & KNI 06 & 1 & 26 & 35 & CTN & SBM & & \\
\hline B. Savoye & KNI 06 & 1 & 35 & 42 & CTN & ARG & & \\
\hline B. Savoye & KNI 06 & 1 & 41 & 42 & CTN & $\mathrm{COQ}$ & & \\
\hline B. Savoye & KNI 06 & 1 & 42 & 47 & CTN & ARG-SBF & & \\
\hline B. Savoye & KNI 06 & 1 & 47 & 50 & CTN & SBF & & \\
\hline B. Savoye & KNI 06 & 1 & 50 & 55 & CTN & $\mathrm{ARG}$ & & \\
\hline B. Savoye & KNI 06 & 1 & 55 & 68 & CTN & SBF & GRN & \\
\hline B. Savoye & KNI 06 & 1 & 68 & 73 & CTI & SBM & & \\
\hline B. Savoye & KNI 06 & 1 & 73 & 78 & CTI & SBG-GRV & & $\begin{array}{l}\text { très grossier } \\
\text { débris coquilles }\end{array}$ \\
\hline B. Savoye & KNI 06 & 1 & 78 & 93 & CTN & SBG & & \\
\hline B. Savoye & KNI 06 & 1 & 73 & 93 & & & GRI & \\
\hline
\end{tabular}

d'une carotte au sein d'un laboratoire, d'une équipe de chantier ou d'une société. Il est possible d'ajouter des lignes de commentaire à chaque saisie, afin de compléter une description qui serait trop succincte avec les simples codes-barres.

\section{CONCLUSION}

Le logmètre est un outil performant d'aide à la saisie des descriptions de carottes. Il est portable, d'un coût modeste et très évolutif. Il ne remplace pas l'expertise du géologue, mais constitue une aide précieuse pour la constitution d'archives numériques et l'interprétation comparée avec d'autres données issues par exemple de bancs de mesures multiparamètres.

Venant après ceux-ci dans la chaîne d'acquisition de données, le logmètre peut s'intégrer dans différents types d'environnement de gestion de carottes.

Remarque: l'utilisation du logmètre est couverte par le brevet $\mathrm{n}^{\circ}$ FR 9110113.

\section{RÉFÉRENCES}

[1] Kerbrat R., Floch G., Savoye B., Le logmètre : un nouvel outil de saisie des descriptions de carotte à partir des codes-barres, IAS-16 ${ }^{\text {th }}$ Regional Meeting of Sedimentology - $5^{\mathrm{e}}$ Congrès français de sédimentologie-ASF, Book of Abstracts, publica-

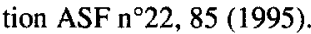

[2] Morizur Y., Ogor A., Lespagnol P., Procédé et dispositif de mesures d'une longueur par codes-barres, et leur application au traitement du poisson, Brevet d'invention INIP $n^{\circ} 9110113$ (1991).
[3] Morizur Y., Ogor A., Lespagnol P., Bar codes in fisheries research : development of the "ichthyometer", Aquat. Living Resour. 7 (1994) 295-300.

[4] Morizur, Y., Comment : Bar-Coded Measuring Systems, Transactions of the American Fisheries Society 124 (1995) 640641.

[5] Sigler M.F., An electronic Measuring Board with Bar Codes. Transactions of the American Fisheries Society 123 (1994) $115-117$. 